\title{
Editorial
}

\section{MRI in head and neck tumours}

\author{
Rabindran $^{1}$, Gedam DS ${ }^{2}$ \\ ${ }^{1}$ Dr. Rabindran, Consultant, Neonatologist, Billroth Hospital, Chennai, India, ${ }^{2}$ Dr D Sharad Gedam, Professor of Pediatrics, \\ RKDF Medical College, Bhopal, MP, India.
}

Address for Correspondence: Dr. Rabindran, E- mail: rabindranindia@yahoo.co.in

\begin{abstract}
Head and neck cancer are prevalent reason for cancer. Compared to CT, MRI has better tissue, spatial and contrast resolution, Non-ionizing radiation, Non-invasiveness, Multiplanar imaging and soft tissue definition.
\end{abstract}

Key words: Head and neck tumours, Functional MRI,Diffusion Weighted -MRI

\section{Introduction}

Head and neck cancer (HNC) includes malignancy of sinonasal region, pharynx, larynx, oral cavity, glands and lymphatic system of neck[1]. Due to excellent soft tissue contrast resolution, Magnetic Resonance Imaging (MRI) is nowadays increasingly being used in evaluating soft tissues like temporomandibular joint disk, soft tissues neoplasms, malignant lymph nodes, marrow oedema, mandibular carcinoma penetration, pre-surgical dental implant assessment and assessment of dental caries, pulp, and periapical structures. Compared to CT, MRI has better tissue, spatial and contrast resolution, Nonionizing radiation, Non-invasiveness, Multiplanar imaging and soft tissue definition. MRI is contraindicated in certain conditions like presence of Vascular clips, Metallic foreign bodies, Coronary and peripheral artery stents, Prosthetic heart valves, Hemodynamic monitoring devices, Permanent cardiac pace-makers, Implantable defibrillators, Permanent contraceptive devices and Cochlear implants.

For HNC, imaging surveillance is done after treatment for detecting residual or recurrent tumour. Interpretation of these post-treatment follow-up imaging is difficult with conventional methods like computed tomography (CT) as surgery alters anatomy and radiation/ chemotherapy causes oedema and fibrosis which mimics tumour recurrence. Functional MRI becomes essential in such situations. Beyond anatomical study using conventional imaging, functional MRI have role in head and neck tumour detection, characterization, staging, treatment response monitoring and prediction [2].

It quantifies tumour characteristics related to tumour physiology and biology. Functional MRI techniques used in HNC include Diffusion Weighted - MRI (DW-MRI), Dynamic contrast-enhanced MR imaging (DCE-MRI), Blood oxygen level-dependent (BOLD) MRI, MR Elastography (MRE), MR spectroscopy (MRS), Spinlock and chemical exchange saturation transfer (CEST) MRI. DW-MRI quantifies diffusion of molecules (typically water), in biological tissues, whereas perfusion MRI assesses passage of blood through tissues. They also can be used to differentiate different tumour types. DW-MRI can be used for risk stratification in thyroid cancer and nodal staging in HNC patients. MRI imaging biomarkers helps inpatient selection for both treatment intensification and de-escalation. DCE-MRI is most frequently used perfusion MRI method to study microstructure, permeability, tumour angiogenesis and hypoxia. DCEMRI sequential MR images are obtained before, during and after a bolus contrast administration.

It quantifies various vascular biomarkers like blood volume, blood flow and permeability. It also identifies early locoregional recurrence, differentiates metastatic lymph nodes from normal nodesand predicts tumour resistance and also serves as an imaging biomarker for hypoxia [3]. DCE- MRI helps in differentiating recurrent malignant salivary gland tumors from post-treatment changes [4]. BOLD MRI analyses changes in magnetic susceptibility of tissue due to paramagnetic deoxy haemoglobin. Decrease in transverse relaxation time T2 indicates increase in deoxy haemoglobin [5]. Rate of transverse relaxation R2 (1/T2) is used as a biomarker for radiation response [6]. It non-invasively analyses neural function and connectivity by measuring hemodynamic response to brain activation.

However DCE-MRI can be affected by various tissue relaxation time, permeability, vascularity, haematocrit and hypoxia. BOLD-MRI only measures T2 weighted MR signal variation due to deoxyhemoglobin, hence it can be affected by blood flow volume. Therefore careful interpretation of functional MRI is mandatory. MRS studies non-invasively the concentration of metabolites resonating at different frequencies from water within the tissue in the form of MR spectrum [7]. Pointed-resolved 


\section{Editorial}

spectroscopy and stimulated echo acquisition modesequence are used to study the spectrum data. MRE is a non-invasive palpation imaging quantitative method that analyses biomechanical properties like elasticity and viscosity by using complex MR images. It can be useful to quantify difficult lesions like fibrosis, a common complication of radiotherapy for HNCs. Spin-lock and CEST MRI are two MRI techniques to analyse chemical exchange in tissues [8].

CEST is a molecular and cellular MRI method which studies in vivo chemical exchange processes between free water and mobile exchangeable exogenous or endogenous agents [9]. It is studied as method to differentiation of brain tumour reoccurrence from radiation induced necrosis [10].

Re-irradiation with MRI guided radiation therapy with advanced technologies like intensity modulated radiation therapy and stereotactic body radiation therapy is helpful in disease control for patients with recurrent and second primary head and neck cancers. Due to medical advancement, newer technologies of imaging have made diagnosis, prognosis, treatment response, therapy and early relapse recognition possible in head and neck tumours.

\section{Abbreviations}

BOLD: Blood Oxygen Level-Dependent, CEST: chemical exchange saturation transfer, CT: Computed Tomography, DCE-MRI: Dynamic Contrast- Enhanced MR Imaging, DW-MRI: Diffusion Weighted-MRI HNC: Head and Neck Cancer, MRE: Magnetic Resonance Elastography, MRI: Magnetic Resonance Imaging MRS: Magnetic Resonance Spectroscopy

\section{Funding: Nil, Conflict of interest: None}

Permission of IRB: Yes

\section{References}

1. Jemal A, Bray F, Center MM, Ferlay J, Ward E, Forman D. Global cancer statistics. CA Cancer J Clin. 2011 Mar-Apr;61(2):69-90. doi: 10.3322/ caac. 20107.

2. Razek AA, Tawfik AM, Elsorogy LG, Soliman NY. Perfusion CT of head and neck cancer. Eur J Radiol. 2014 Mar; 83 (3):537-44. doi: 10.1016/j. ejrad. 2013. 12.008. Epub 2013 Dec 16.
3. Gaddikeri S, Gaddikeri RS, Tailor T, Anzai Y. Dynamic Contrast-Enhanced MRImaging in Head and Neck Cancer: Techniques and Clinical Appli-cations. AJNR Am J Neuroradiol.2016 Apr; 37(4): 588-95. doi: 10. 3174/ ajnr.A4458. Epub 2015 Oct 1.

4. SiezaSamir, Mohamed AliEl-Adalany, Emad EldeenHamed. Value of dynamic contrast enhanced magnetic resonance imaging in the differentiation between post-treatment changes and recurrent salivary gland tumors. The Egyptian Journal of Radiology and Nuclear Medicine. Volume 47, Issue 2, June 2016, Pages 477-486. https://doi.org/ 10.1016/j. ejrnm.2016.01.005

5. Krishna MC, Subramanian S, Kuppusamy P, Mitchell JB. Magnetic resonance imaging for in vivo assessment of tissue oxygen concentration. SeminRadiatOncol. 2001 Jan;11(1):58-69.

6. Li XS, Fan HX, Fang H, Song YL, Zhou CW. Value of $\mathrm{R} 2 *$ obtained from $\mathrm{T} 2 *$-weighted imaging in predicting the prognosis of advanced cervical squamous carcinoma treated with concurrent chemoradiotherapy. J Magn Reson Imaging. 2015 Sep; 42 (3):681-8. doi: 10. 1002/ jmri.24837.

7. Castillo M, Kwock L, Mukherji SK. Clinical applications of proton MR spectroscopy. AJNR Am J Neuroradiol. 1996 Jan;17(1):1-15.

8. Markkola AT, Aronen HJ, Lukkarinen S, Ramadan UA, Tanttu JI, Sepponen RE. Multiple-slices pin lock imaging of head and necktumors at 0.1Tesla: exploring appropriate imaging parameters with reference to T2weightedspin-echotechnique. Invest Radiol.2001 Sep;36 (9): 531-8.

9. Ward KM, Aletras AH, Balaban RS. A newclass of contrast agents for MRIbased on proton chemical exchange dependent saturation transfer (CEST). J Magn Reson. 2000 Mar;143(1):79-87.

10. Zhou J, Tryggestad E, Wen Z, Lal B, Zhou T, Grossman R, Wang S, Yan K, Fu DX, Ford E, Tyler B, Blakeley J, Laterra J, van ZijlPC. Differentiation between glioma and radiation necrosisusing mole cular magnetic resonance imaging of endogenousproteins and peptides. Nat Med. 2011Jan;17(1):130-4. doi: 10.1038/nm.2268. Epub 2010 Dec 19.

\section{How to cite this article?}

Rabindran, Gedam DS. MRI in head and neck tumours. Int J Med Res Rev 2017;5 (12):974-975.doi:10.17511/ijmrr. 2017.112.01 